\title{
A Case Report of Cardiac Amyloidosis Presenting with Chest Discomfort
}

\author{
Binay Kumar Adhikari, Yonggang Wang, Bo Li, Quan Liu, Weihua Zhang* \\ Center of Cardiovascular Medicine, The First Hospital of Jilin University, Changchun, China \\ Email: *weihua@jlu.edu.cn
}

How to cite this paper: Adhikari, B.K., Wang, Y.G., Li, B., Liu, Q. and Zhang, W.H. (2018) A Case Report of Cardiac Amyloidosis Presenting with Chest Discomfort. World Journal of Cardiovascular Diseases, 8, 162-168.

https://doi.org/10.4236/wjcd.2018.82016

Received: January 15, 2018

Accepted: February 10, 2018

Published: February 13, 2018

Copyright () 2018 by authors and Scientific Research Publishing Inc. This work is licensed under the Creative Commons Attribution International License (CC BY 4.0).

http://creativecommons.org/licenses/by/4.0/

\begin{abstract}
Introduction: Cardiac amyloidosis is one of the several systemic manifestations of amyloidosis. It is a relatively uncommon disease. Patients with cardiac amyloidosis clinically present with rapidly progressive heart failure. Case Presentation: We present a case of 64-year-old man with multiple episodes of chest discomfort accompanied sometimes with dyspnoea for the past several months. A non-specific ST-T changes with low voltage limb leads on ECG, a diastolic dysfunction with preserved ejection fraction on echocardiography, increased troponin and BNP, increased serum $\lambda / \kappa$ light chain ratio led us to highly suspect amyloidosis with cardiac involvement. Abdominal fat pad biopsy confirmed the amyloid type change of subcutaneous fat, with immunohistochemistry revealing Lambda positive. After confirmed diagnosis of primary systemic amyloidosis with cardiac involvement, patient completed six cycles of chemotherapy with bortezomib and dexamethasone. The patient showed significant improvements after chemotherapy and supportive therapy. Conclusion: Amyloidosis is relatively fatal disease. Patients often have a short life expectancy after symptom onset. Early diagnosis is crucial to proper management and better prognosis.
\end{abstract}

\section{Keywords}

Cardiac Amyloidosis, Cardiomyopathy, Heart Failure

\section{Introduction}

Amyloidosis is a multisystem disorder that results from extracellular deposition of insoluble amyloid protein in various organs. The most commonly affected organs are heart and kidney. The heart is involved in up to $50 \%$ of systemic primary amyloidosis [1], clinically presents with progressive dyspnoea and peripheral oedema [2]. Cardiac involvement of amyloidosis has major impact on 
prognosis [3], with a median survival less than 6 months without treatment. Endomyocardial biopsy is gold standard for diagnosing cardiac amyloidosis. However, it is more invasive and much difficult to perform. Hence, abdominal fat pad biopsy is preferred over endomyocardial biopsy to establish definite diagnosis of systemic amyloidosis [4]. We here present a case of primary systemic amyloidosis with cardiac involvement and clinical manifestation of multiple episodes of chest discomfort.

\section{Case Report}

A 64-year-old male smoker presented to our hospital with complains of multiple episodes of chest discomfort which sometimes is accompanied with dyspnoea mostly occurring at night time, symptoms relieved upon sitting in an upright position. The patient also reported a 40 years history of bronchitis. Physical examination showed a pulse rate $62 / \mathrm{min}$, BP $107 / 70 \mathrm{mmHg}$, normal jugular venous pulse, enlarged left cardiac border on percussion, normal heart sounds, clear lung fields and mild pitting oedema of bilateral lower limbs. Relevant blood investigations revealed $\mathrm{RBC} 3.78 \times 10^{12} / \mathrm{L}$, hemoglobin $116 \mathrm{~g} / \mathrm{L}$, platelet $264 \times$ $10^{9} / \mathrm{L}$, urea $11.62 \mathrm{mmol} / \mathrm{L}$, creatinine $96.7 \mathrm{umol} / \mathrm{L}$, sodium $133.2 \mathrm{mmol} / \mathrm{L}$, chlorine: $96.1 \mathrm{mmol} / \mathrm{L}$, AST $16.4 \mathrm{U} / \mathrm{L}$, ALT $19.5 \mathrm{U} / \mathrm{L}$, total protein $51.6 \mathrm{~g} / \mathrm{L}$, albumin $30.8 \mathrm{~g} / \mathrm{L}$, globulin $20.7 \mathrm{~g} / \mathrm{L}$, pro-albumin $0.17 \mathrm{~g} / \mathrm{L}, \mathrm{BNP} 3420 \mathrm{pg} / \mathrm{ml}$ and troponin I $0.196 \mathrm{ng} / \mathrm{mL}$. On protein electrophoresis, total protein $57.1 \mathrm{~g} / \mathrm{L}$, albumin $59.6 \%, \alpha 1$ globulin $3.8 \%, \alpha 2$ globulin $9.4 \%, \beta$ globulin $10.4 \%$ and $\gamma$ globulin $16.8 \%, \beta 2$ microglobulin $3.47 \%$. Serum immunofixation electrophoresis showed non-significant level of IgD, IgA, IgG, IgM, $\lambda$ light chain and $k$ light chain. Serum free $\lambda$ chain was $296.00 \mathrm{mg} / \mathrm{L}$ and free $\kappa$ chain was $19.80 \mathrm{mg} / \mathrm{L}$, thus, serum $\lambda / \kappa$ ratio was $15: 1$. The urine $\lambda$ light chain $5.50 \mathrm{mg} / 24 \mathrm{~h}$, urine $\kappa$ light chain 15.90 $\mathrm{mg} / 24 \mathrm{~h}$. Serological tests resulted rheumatoid factor $11.50 \mathrm{IU} / \mathrm{mL}$, ASO 65.40 $\mathrm{IU} / \mathrm{mL}$, hypersensitive CRP $3.02 \mathrm{mg} / \mathrm{L}$, complement C3 0.97, and complement C4 0.24. Screening and confirmatory tests for antinuclear antibody and anti-neutrophil antibody, and thyroid function had no abnormalities.

ECG showed non-specific ST-T changes with low voltage limb leads (Figure 1). Holter ECG revealed sinus bradycardia, occasional atrial extrasystole-couplet type, and paroxysmal atrial tachycardia. Echocardiography showed left atrial (46 $\mathrm{mm})$ and left ventricular $(58 \mathrm{~mm})$ enlargement, along with increased thickness of basal interventricular septum (12 mm), segmental wall motion abnormality of left ventricle, E peak $83 \mathrm{~cm} / \mathrm{s}$; A peak $111 \mathrm{~cm} / \mathrm{s}$; TDI: E/e' > 15 with preserved ejection fraction (58\%), aortic valve calcification and mild regurgitation of both mitral and aortic valve. Lung multi-slice CT had features of bronchitis with inflammatory changes and localized emphysema of bilateral lungs, thoracic aortic calcification and coronary artery sclerosis (Figure 2). Venous ultrasound of lower extremities had findings of subcutaneous oedema. Bone marrow aspiration and biopsy reported increased number of erythoid cells, plasmablasts accounting $1 \%$, and presence of Mott cells. Abdominal fat pad biopsy confirmed 


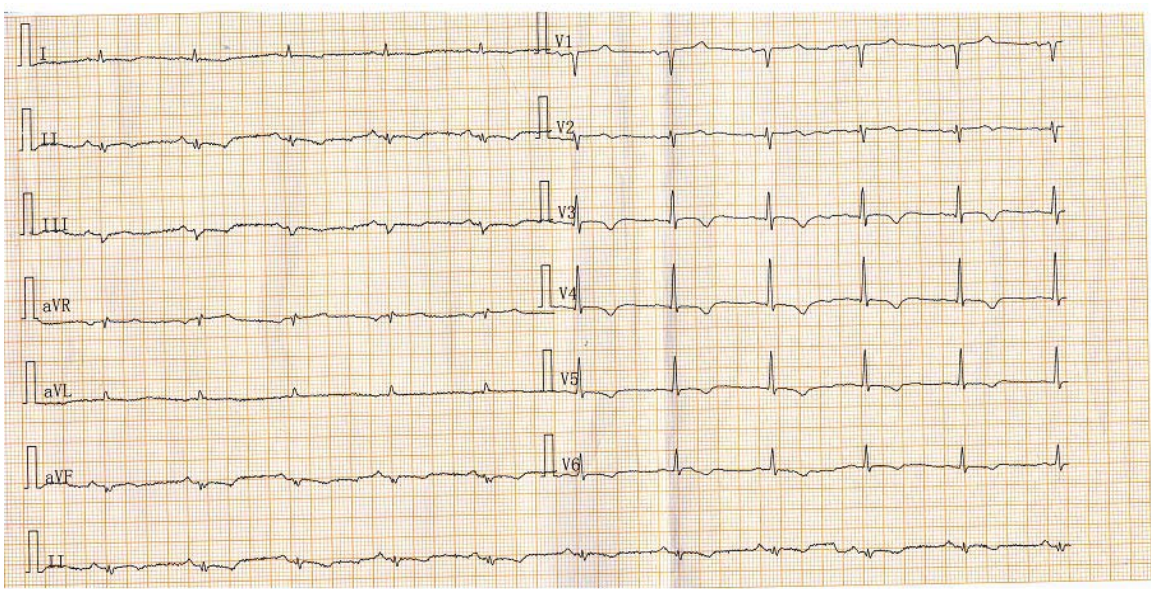

Figure 1. ECG reveals non-specific ST-T changes with low voltage limb leads.

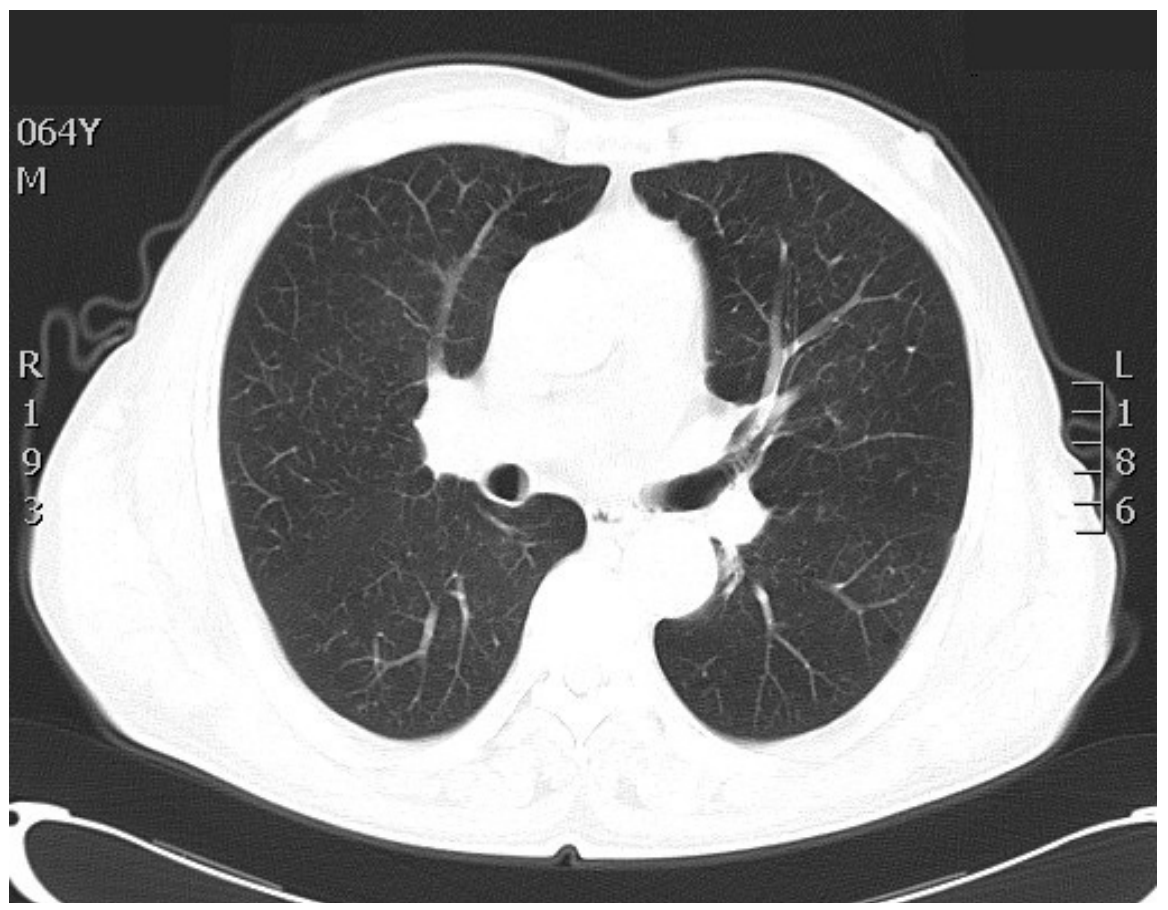

Figure 2. Lung multi-slice CT showed bronchitis with inflammatory changes in both lungs and localized bilateral pulmonary emphysema, thoracic aortic calcification and coronary artery sclerosis.

the amyloid type change of subcutaneous tissue (Figure 3). Similarly, immunohistochemistry revealed Lambda positive, and apple green birefringence on staining with Congo red. After patient was diagnosed with systemic primary amyloidosis with cardiac involvement, patient was admitted to haematology department for chemotherapy. So far a total of 6 cycles of chemotherapy with bortezomib and dexamethasone have already performed. Bortezomib was administered $1.3 \mathrm{mg} / \mathrm{m}^{2}$ subcutaneously per day on days $1,4,8$, and 11 every 21 days and dexamethasone $10 \mathrm{mg}$ intravenously once a day on days $1-2,4-6,8-9$, and 11 - 12. Other supportive therapies included fluid and salt restriction, 

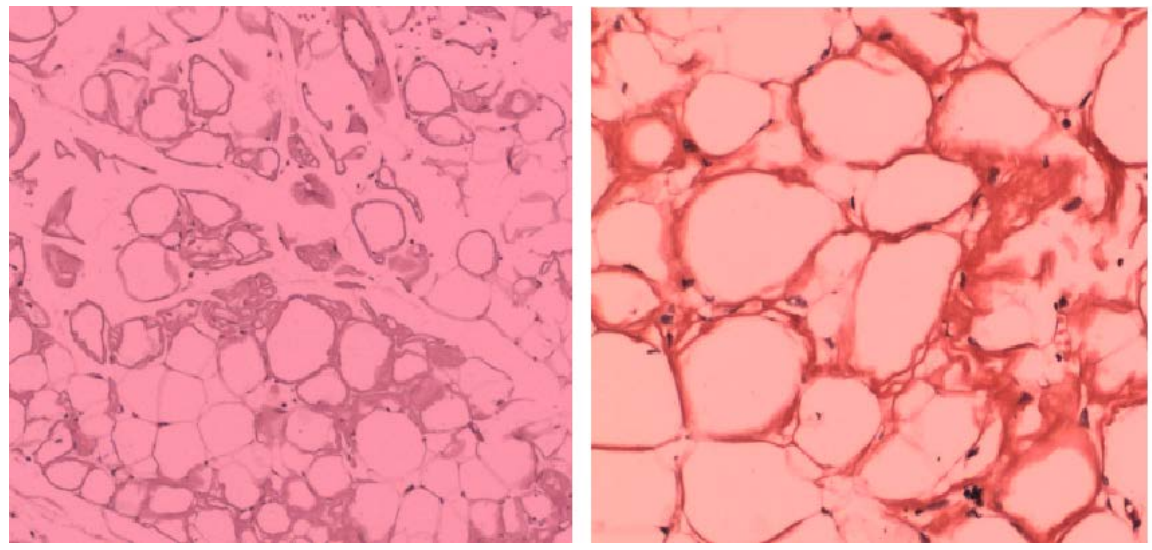

Figure 3. Abdominal fat pad biopsy revealed amyloid type changes of subcutaneous fat immunohistochemisty: Lambda (weak + ); Congo $(+)$.

diuretics and spironolactone. Repeated measurement of serum free $\lambda$ chain (62 $\mathrm{mg} / \mathrm{L})$, serum free $k$ chain $(24.9 \mathrm{mg} / \mathrm{L})$, troponin $\mathrm{I}(0.161 \mathrm{ng} / \mathrm{mL})$ and serum BNP $(2060 \mathrm{pg} / \mathrm{mL})$ was performed after $1^{\text {st }}$ cycle of chemotherapy which showed decreasing tendency comparing with baseline results. Following up after 6 months of chemotherapy and other supportive measures, patient had significant symptomatic relief with chest discomfort and dyspnoea. The peripheral edema was also reduced compared with presentation at admission.

\section{Discussion}

Amyloidosis is a multisystem disorder due to deposition of insoluble amyloid fibrils that have characteristic $\beta$-pleated sheet configuration and produces apple-green birefringence under polarized light when stained with Congo red dye [5]. The type of amyloidosis is defined by precursor protein. The most common amyloidosis is primary systemic amyloidosis, also called amyloid light chain (AL) amyloidosis, is caused when a person's antibody producing cells (plasma cells) do not function properly and produce abnormal protein fibres made of components of antibodies called light chains [6]. Hence, it is often associated with other blood disorders, such as multiple myeloma and Waldenstrom's macroglobulinemia.

Amyloid deposition in the heart results in cardiac amyloidosis, clinically presents with rapidly progressive heart failure because of restrictive cardiomyopathy, and sometimes presents with typical angina due to amyloid deposition in arterial vessel wall causing progressive luminal narrowing and hence myocardial ischemia and impairment [7]. Anish et al. described a case with cardiac amyloidosis presenting as recurrent acute coronary syndrome without coronary artery obstruction [8]. Diagnosis of amyloidosis relies on clinical awareness of the disease, clinical features, blood and tissue analysis, and positive findings on biopsy. For patients with heart involvement, the most common clinical feature is diastolic heart failure, which is manifested as progressive dyspnea. In our case, patient complained of multiple episodes of chest discomfort from last 4 months, 
occurring multiple times a day, sudden onset without any clear precipitating factor, getting relieved after few minutes of sitting upright, sometimes accompanied with dyspnoea and mostly occurring during night time. Patient's chest pain had no relation with exertion or exercise. Later findings of electrocardiography, transthoracic echocardiography, serum cardiac biomarkers, and serum protein electrophoresis lead to highly suspicion for amyloidosis that was confirmed with abdominal fat pad biopsy and immunohistochemisty. Some cases have also reported pharyngeal symptoms related to amyloidosis causing dysphagia [9]. So the clinicians should pay more attention to the systemic disorders such as nephropathy, skin damage, macroglossia, sympathetic or vagus nerve disease e.g. constipation or orthostatic hypotension to recognize amyloidosis. For differentiating with secondary causes of amyloidosis tumor markers, rheumatoid factor, anticuclear antibodies, autoantiboidies tests were also performed, all tests found negative. Although ${ }^{99 \mathrm{~m}} \mathrm{Tc}$ DPD scintigraphy, and genetic testing were not done which are useful in differentiating AL with transthyretin-related amyloidosis and senile systemic amyloidosis. We performed myelogram and bone marrow biopsy but did not reveal monoclonal plasma cells, which is only found in $10 \%-15 \%$ of patients [10].

ECG frequently shows low voltage limb leads. First degree AV block is common and Q waves are frequently seen in leads V1 to V3. In cardiac amyloidosis, echocardiography generally reveals a pattern suggestive of infiltrative cardiomyopathy. In our patient, echocardiography revealed enlarged left atrium and left ventricle because of regurgitation present in both mitral and aortic valve. Measurement of serum free $\kappa$ chain and $\lambda$ chain demonstrates an excess of $\lambda$ chain in our case. For other systemic manifestation of amyloidosis, abdominal fat pad biopsy was performed, which demonstrated apple green birefringence on staining with Congo red, and $\lambda$ positive on immunohistochemistry. Cardiac MRI is also useful for diagnosis, and is preferred because of its non-invasive nature prior to any invasive procedure [11]. Troponin and BNP/NT-proBNP reflects high filling pressures, and are highly useful in diagnosis and assessment of prognosis of AL amyloidosis [12]. According to Mayo Clinic staging, patients are stratified into three groups; high risk group if both biomarkers are increased, intermediate risk group if at least one biomarker is above the cut off value and low risk if both biomarkers are below cut off values [13]. In our case, we found highly increased BNP and troponin at diagnosis, which indicated we were dealing with a patient of high risk. Based on Mayo Clinic staging, those in high risk group have median survival of 7 - 8 months.

Management of systemic amyloidosis is done by stopping the production of abnormal light chains by plasma cells and supportive care provided to decrease symptoms and support organ function [14]. Chemotherapy strategy includes dexamethasone with either melphalan or cyclophosphamide or an immunomodulatory (thalidomide, lealidomide or proteasome inhibitor bortezomib) [15]. The response to treatment is assessed by measuring free light chains in the 
blood, which return to normal in successful treatment. Regardless of recent advances in management, long term prognosis of cardiac amyloidosis is poor, especially if diagnosis is delayed [16]. Several cases reported by Chandrashekhar et al concluded that despite intensive chemotherapy, cardiac treatment is supportive and the results are often disappointing [17]. In our case, after starting chemotherapy, patient's heart failure symptoms relieved. The serum free light chain level was also decreased. It has been more than six months of confirmed diagnosis and patient condition has significant improvement.

\section{Conclusion}

Amyloidosis is uncommon, yet relatively fatal disease. In many patients, it is underdiagnosed or unrecognized. This results in increased morbidity and decreased life expectancy. Hence, early diagnosis is crucial to proper management and better prognosis. Since, prognosis is very poor, if diagnosis is delayed. Early institution of chemotherapy along with other supportive measures stabilizes amyloidogenic substance in blood, improves patient condition and prolongs life.

\section{Funding}

This study was supported by grant from Jilin Province Science and Technology Project (grant No. 20170520012JH) and National Natural Science Foundation (grant No. 81400279).

\section{Acknowledgements}

We especially thank patients and their relatives for allowing us to share their information to all readers of medical society.

\section{References}

[1] Halwani, O. and Delgado, D.H. (2010) Cardiac Amyloidosis: An Approach to Diagnosis and Management. Expert Review of Cardiovascular Therapy, 8, 1007-1013. https://doi.org/10.1586/erc.10.41

[2] Kappor, P., Thenappan, T., Singh, E., Kumar, S. and Greipp, P.R. (2011) Cardiac Amyloidosis; A Practical Approach to Diagnosis and Management. The American Journal of Medicine, 124, 1006-1015. https://doi.org/10.1016/j.amjmed.2011.04.013

[3] Mohty, D., Damy, T., Cosnay, P., Echahidi, N., Casset, S.D., Virot, P. and Jaccard, A. (2013) Cardiac Amyloidosis: Updates in Diagnosis and Management. Archives of Cardiovascular Disease, 106, 528-540. https://doi.org/10.1016/j.acvd.2013.06.051

[4] Crotty, T.B., Li, C.Y., Edwards, W.D. and Suman, V.J. (1995) Amyloidosis and Endomyocardial Biopsy: Correlation and Extent and Pattern of Deposition with Amyloid Immunophenotype in 100 Cases. Cardiovascular Pathology, 4, 39-42. https://doi.org/10.1016/1054-8807(94)00023-K

[5] Desai, H.V., Aronow, W.S., Peterson, S.J. and Frishman, W.H. (2010) Cardiac Amyloidosis: Approaches to Diagnosis and Management. Cardiology in Review, 18, 1-11. https://doi.org/10.1097/CRD.0b013e3181bdba8f

[6] Falk, R.H, Alexander, K.M, Liao, R. and Dorbala, S. (2016) AL (Light-Chain) Cardiac Amyloidosis: A Review of Diagnosis and Therapy. Journal of the American 
College of Cardiology, 68, 1323-1341.

https://doi.org/10.1016/j.jacc.2016.06.053

[7] Sanchorawala, V. (2006) Light-Chain (AL) Amyloidosis: Diagnosis and Treatment. Clinical Journal of the American Society of Nephrology, 1, 1331-1341. https://doi.org/10.2215/CJN.02740806

[8] Anish, G. and Brain, M.C. (2015) Cardiac Amyloidosis Presenting as Recurrent Acute Coronary Syndrome with Unobstructed Coronary Arteries: Case Report. Indian Heart Journal, 67, 570-573. https://doi.org/10.1016/j.ihj.2015.08.029

[9] Masafumi, O. and Shigeru, K. (2018) Dysphagia due to Systemic Light Chain Amyloidosis revealed by Videoendoscopic and Videofluorosgraphic Swallowing Examinations. Otolaryngology Case Reports, 6, 406.

[10] Rapezzi, C., Quarta, C.C., Riva, L., Longhi, S., Gallelli, I., Lorenzini, M., et al. (2010) Transthyretin-Related Amyloidosis and the Heart: A Clinical Overview. Nature Reviews Cardiology, 7, 398-408. https://doi.org/10.1038/nrcardio.2010.67

[11] Maceira, A.M., Joshi, J., Prasad, S.K., Moon, J.C., Perugini, E., Harding, I., et al. (2005) Cardiovascular Magnetic Resonance in Cardiac Amyloidosis. Circulation, 111, 186-193. https://doi.org/10.1161/01.CIR.0000152819.97857.9D

[12] Dispenzieri, A., Gertz, M.A., Kyle, R.A., Lacy, M.Q., Burritt, M.F., Therneau, T.M., et al. (2004) Serum Cardiac Troponins and N-Terminal pro-Brain Natriuretic Peptide: A Staging System for Primary Systemic Amyloidosis. Journal of Clinical Oncology, 22, 3751-3757. https://doi.org/10.1200/JCO.2004.03.029

[13] Gertz, M.A., Comenzo, R., Falk, R.H., Fermand, J.P., Hazenberg, B.P., Hawkins, P.N., et al. (2005) Definition of Organ Involvement and Treatment Response in Immunoglobulin Light Chain Amyloidosis (AL): A Consensus Opinion from the $10^{\text {th }}$ International Symposium on Amyloid and Amyloidosis, Tours, France, 18-22 April. American Journal of Hematology, 79, 319-328. https://doi.org/10.1002/ajh.20381

[14] Cristina, C.Q., Jenna, L.K. and Rodney, H.F. (2012) Cardiac Amyloidosis. Circulation, 126, e178-e182. https://doi.org/10.1161/CIRCULATIONAHA.111.069195

[15] Selvanayagam, J.B., Hawkins, P.N., Paul, B., Myerson, S.G. and Neubauer, S. (2007) Evaluation and Management of the Cardiac Amyloidosis. Journal of the American College of Cardiology, 50, 2101-2110. https://doi.org/10.1016/j.jacc.2007.08.028

[16] Gertz, M.A. (2011) Immunoglobulin Light Chain Amyloidosis: 2011 Update on Diagnosis, Risk Stratification, and Management. American Journal of Hematology, 86, 181-186. https://doi.org/10.1002/ajh.21934

[17] Munjewar, C., Agrawal, R. and Sharma, S. (2014) Cardiac Amyloidosis: A Report of Two Cases. Indian Heart Journal, 66, 473-476. https://doi.org/10.1016/j.ihj.2014.05.028 